\title{
Repercussões da pandemia de COVID-19 na relação das mães com o aleitamento
}

\section{materno}

\author{
Repercussions of the COVID-19 pandemic on mothers relationship with breastfeeding \\ Repercusiones de la pandemia de COVID-19 en la relacion de las madres con la lactancia materna
}

Recebido: 03/02/2022 | Revisado: 10/02/2022 | Aceito: 17/02/2022 | Publicado: 25/02/2022

\author{
Mariana Mylena Melo da Silva \\ ORCID: https://orcid.org/0000-0003-3714-9813 \\ Centro Universitário Estácio do Recife, Brasil \\ E-mail: marianamylena@hotmail.com \\ Júlia Kauana Fernandes Moreira \\ ORCID: https://orcid.org/0000-0002-5755-8377 \\ Centro Universitário Estácio de Recife, Brasil \\ E-mail: juliakauanafm@ hotmail.com \\ João Victor Lopes Oliveira \\ ORCID: https://orcid.org/0000-0001-8177-7388 \\ Centro Universitário Estácio do Recife, Brasil \\ E-mail: victorlopes0029@gmail.com \\ Roumayne Medeiros Ferreira Costa \\ ORCID: https://orcid.org/0000-0003-0437-0714 \\ Centro Universitário Estácio do Recife \\ E-mail: Roumayne@ hotmail.com
}

\section{Resumo}

A pandemia de COVID-19 está relacionada a uma doença respiratória aguda, causada pelo novo coronavírus (SARSCoV-2), de evolução ainda pouco conhecida. As atuais recomendações, no que diz respeito a amamentação, é a manutenção do aleitamento materno, até mesmo para as mães que apresentam suspeita ou confirmação de COVID-19. Objetivou-se Avaliar as repercussões da pandemia da COVID-19 na relação das mães com o aleitamento materno. Tratando-se de um estudo transversal, exploratório, descritivo, com abordagem quantitativa, realizado na Unidade de Saúde da Família Liberdade, localizada no município de Tracunhaém, situado na Zona da Mata Norte de Pernambuco. A amostra foi composta por 78 puérperas que participaram das consultas de pré-natal na unidade e que quiseram participar da pesquisa, já a coleta de dados foi realizada no período de agosto a setembro de 2021. A pesquisa, inicialmente, contou com 92 (100\%) puérperas cujo contato foi cedido pela USF analisada, das quais 14 (15\%) não se disponibilizaram a participar da pesquisa e/ou não atenderam às ligações telefônicas ou mudaram de número, totalizando 78 puérperas (100\%) participantes. Em relação aos dados discutidos, observou-se que a maioria das puérperas não recebeu orientação quanto aos cuidados durante a amamentação, podendo, assim, aumentar o risco de contaminação da criança.

Palavras-chave: Aleitamento materno; Saúde materno-infantil; Infecção por Coronavírus.

\begin{abstract}
The COVID-19 pandemic is related to an acute respiratory disease caused by the new coronavirus (SARS-Cov-2), an evolution still little known. The current recommendations, regarding breastfeeding, is the maintenance of breastfeeding, even for mothers who present suspicion or confirmation of COVID-19. Objective to evaluate the repercussions of the COVID-19 pandemic on the relationship between mothers and breastfeeding. Method: This is a cross-sectional, exploratory, descriptive study with a quantitative approach, conducted at the Liberdade Family Health Unit, located in the city of Tracunhaém, in the Zone of North Forest of Pernambuco. The sample was composed of 78 postpartum women who participated in prenatal consultations in the unit, and who wanted to participate in the research, while data collection was carried out in the period from August to September 2021. Results and discussion: The survey, initially, had $92(100 \%)$ postpartum women whose contact was provided by the analyzed FHU, of which $14(15 \%)$ were not available to participate in the survey and/or did not answer the phone calls or changed their number, totaling 78 postpartum women (100\%) participants. Conclusion: In relation to the data discussed, it was observed that the majority of postpartum women did not receive guidance regarding care during breastfeeding, thus increasing the risk of contamination of the child.
\end{abstract}

Keywords: Breastfeeding; Maternal and child health; Coronavirus infection.

\section{Resumen}

La pandemia de COVID-19 está relacionada con una enfermedad respiratoria aguda provocada por el nuevo coronavirus (SARS-CoV-2), cuya evolución aún es poco conocida. Las recomendaciones actuales, con respecto a la 
lactancia materna, es mantener la lactancia, incluso para las madres con sospecha o confirmación de COVID-19. El objetivo fue evaluar las repercusiones de la pandemia de COVID-19 en la relación entre las madres y la lactancia materna Tracunhaém, ubicada en la Zona da Mata Norte de Pernambuco. La muestra estuvo conformada por 78 puérperas que participaron de consultas de prenatal en la unidad y que quisieron participar de la investigación, ya que la recolección de datos se realizó de agosto a septiembre de 2021. La investigación contó inicialmente con 92 (100\%) puérperas cuyo contacto fue proporcionadas por las USF analizadas, de las cuales 14 (15\%) no estaban disponibles para participar de la investigación y/o no respondieron las llamadas telefónicas o cambiaron de número, totalizando 78 puérperas $(100 \%)$ participantes. En cuanto a los datos discutidos, se observó que la mayoría de las puérperas no recibieron orientaciones sobre los cuidados durante la lactancia, lo que puede, por lo tanto, aumentar el riesgo de contaminación del niño.

Palabras clave: Amamantamiento; Salud maternal e infantil; Infección por coronavirus.

\section{Introdução}

A pandemia de COVID-19 está relacionada a uma doença respiratória aguda causada pelo novo coronavírus (SARSCoV-2), de evolução ainda pouco conhecida. Em 31 de dezembro de 2019, o escritório da Organização Mundial da Saúde (OMS) na China recebeu alerta epidemiológico sobre casos de uma pneumonia com causa desconhecida, na cidade de Wuhan, na província de Hubei. De acordo com Silva et al. (2021), em 7 de janeiro de 2020, pesquisadores chineses isolaram uma cepa SARS-COV-2, da família coronavírus, que ainda não havia sido detectada em humanos. Em 11 de fevereiro de 2020 , o vírus foi oficialmente denominado coronavírus, sendo ele o causador da COVID-19.

Baseado no que dizem Martins et al. (2021), humanos podem ser infectados pelo SARS-CoV-2 por meio de gotículas respiratórias, ou por meio do contato com objetos contaminados pelo vírus. Durante as fases iniciais da epidemia, a infecção foi disseminada, essencialmente, pelo contato de pessoas na comunidade, tendo ocorrido, nesse estágio, quase que exclusivamente em adultos. Depois dessa fase inicial, em meados de janeiro de 2020, passou a ser transmitido também nos núcleos familiares, com disseminação para crianças e idosos.

Tacla et al. (2020) dissertam que o SARS-CoV-2 ainda não foi detectado no leite materno (LM) das mães com confirmação de COVID-19, mas anticorpos específicos contra o mesmo já foram encontrados em amostras de leite de mães infectadas. Foram detectados anticorpos IgG e IgA para SARS-CoV-2 no leite materno, indicando potencial de proteção imunológica em neonatos.

O aleitamento materno (ALM) tem uma ampla importância, e entre os benefícios dessa prática destaca-se o aumento da sobrevivência, por meio da promoção da saúde, e no desenvolvimento da criança. No leite materno encontram-se fatores com atividade antimicrobiana e imunomoduladora, como enzimas, citocinas, componentes do sistema complemento, oligossacarídeos, nucleotídeos, lipídios e hormônios que contribuem para a imunidade e a maturação do sistema imunológico do Recém-nascido (RN). Parafraseando o que afirmam Fonseca et al. (2021), a partir dos dados apresentados pela OMS, o leite humano é o alimento mais completo para a criança, sendo recomendado o aleitamento materno exclusivo, até os 6 meses de idade, que deverá ser complementado até os dois anos ou mais.

De acordo com Melo et al. (2020), ainda não há, na literatura atual, evidências consistentes que sustentem a transmissão vertical do vírus SARS-CoV-2, no período neonatal, através da amamentação. Entretanto, algumas diretrizes nacionais e internacionais, elaboradas antes de 23 de junho de 2020, recomendam que nos casos de mãe infectada, ou com suspeita de COVID-19, o ALM e o contato pele a pele não devem ser realizados imediatamente ao nascer, mas somente após as primeiras medidas de higiene.

Segundo a Sociedade Portuguesa de Neonatologia (2020), para a mãe que tem suspeita de infecção por SARS-CoV-2, recomendam-se medidas extremas de isolamento (higiene das mãos e máscara facial) para amamentar o recém-nascido ou fazer a extração do leite, tomando as precauções de isolamento máximo (higiene das mãos e máscara), sendo este administrado ao recém-nascido por um cuidador saudável. Outra recomendação é que a extração mecânica de LM pode ser uma alternativa 
nas mães com doenças graves, nestes casos, este deve ser administrado por copo, colher, seringa ou biberão. Para isso, a mãe deve lavar as mãos, rigorosamente, antes de bombear e usar a máscara. É importante salientar que, durante o bombeamento, se possível, o equipamento de extração deve ser cuidadosamente limpo por uma pessoa saudável.

É válido ressaltar que a decisão final sobre realizar ou não a amamentação deve ser acordada entre a mãe e a equipe de saúde envolvida, com base no conhecimento científico disponível em cada momento e no estado de saúde da mulher e do recém-nascido, bem como afirmam Melo et al. (2020). As atuais recomendações, no que diz respeito à amamentação, é a manutenção do ALM, até mesmo para as mães que apresentam suspeita ou confirmação de COVID-19. Partindo desse pressuposto, surgiu a seguinte pergunta norteadora do estudo: Quais as repercussões da pandemia da COVID-19 na relação de mães com o aleitamento materno? Portanto, o objetivo deste estudo é avaliar as repercussões da pandemia da COVID-19 na relação de mães com o aleitamento materno.

\section{Metodologia}

Essa respectiva pesquisa tratou-se de um estudo de corte transversal, exploratório, descritivo e de natureza quantitativa realizado na Unidade de Saúde da Família Liberdade, localizada no município de Tracunhaém, situado na Zona da Mata Norte de Pernambuco. A amostra foi composta por 78 puérperas que realizaram o pré-natal na unidade e que quiseram participar da pesquisa, segundo os critérios de elegibilidade. A Unidade de Saúde é composta por 1 equipe, com 1 enfermeiro e 6 Agentes Comunitários de Saúde (ACS) responsáveis, cada um, por, em média, de 180 a 250 famílias, sendo a população coberta de, aproximadamente, 7.000 usuários.

A coleta de dados foi realizada no período de agosto a setembro de 2021, após a aprovação do estudo pelo Comitê de Ética e Pesquisa (CEP) do Centro Universitário Estácio do Recife, com parecer: 4.934.114 e CAAE: 49187021.1.0000.5640. O instrumento utilizado para coleta de dados da pesquisa se deu com base numa entrevista estruturada com 11 perguntas objetivas, elaboradas pelas autoras, a partir de referencial teórico acerca do tema.

O contato aconteceu, inicialmente, com o enfermeiro da unidade, onde o mesmo solicitou a autorização das puérperas, requerendo a assinatura do Termo de Consentimento Livre e Esclarecido (TCLE) para que participassem da pesquisa e repassou os contatos telefônicos aos pesquisadores. Então, os entrevistadores entraram em contato com as mães, por meio de ligação telefônica, para que respondessem às perguntas previamente elaboradas. Dessa forma, ficou assegurada a confidencialidade das entrevistadas. Os resultados foram tabulados e apresentados através de dados estatísticos simples de frequência absoluta e relativa, em forma de tabelas, além disso, foram analisadas, descritivamente, com embasamento teóricocientífico de acordo com os estudos de Pereira et al, (2018); Lüdke e André, (2013); Oliveira et al, (2021).

\section{Resultados e Discussão}

A pesquisa, inicialmente, contou com 92 (100\%) puérperas, cujo contato foi cedido pela USF analisada, das quais 14 (15\%) não se disponibilizaram a participar da pesquisa e/ou não atenderam às ligações telefônicas ou mudaram de número, o que, em resumo, totalizou 78 puérperas $(100 \%)$ participantes que serão descritas nas tabelas a seguir. 
Tabela 1 - Porcentagem das puérperas entrevistadas segundo dados sociodemográficos. Tracunhaém- PE, USF

\begin{tabular}{|l|l|}
\hline Faixa etária & $\%$ \\
\hline 18 a 20 anos & $82 \%$ \\
\hline & $12 \%$ \\
\hline 32 a 31 anos 42 anos & $6 \%$ \\
\hline Total & $100 \%$ \\
\hline & \\
\hline Etnia/raça autodeclarada & \\
\hline Negra & $70,5 \%$ \\
\hline Branca & $23,5 \%$ \\
\hline Parda & $6 \%$ \\
\hline Total & $100 \%$ \\
\hline & \\
\hline Estado civil & $10 \%$ \\
\hline Solteira & $76,5 \%$ \\
\hline Casada & $17,5 \%$ \\
\hline União estável & $6 \%$ \\
\hline Total & $100 \%$ \\
\hline & \\
\hline Escolaridade & $35 \%$ \\
\hline Ensino Médio completo & $12 \%$ \\
\hline Ensino Médio incompleto & \\
\hline Ensino Fundamental completo & \\
\hline Ensino Fundamental incompleto & \\
\hline Ensino Superior incompleto & \\
\hline Total & \\
\hline
\end{tabular}

Fonte: Entrevista (2021).

De acordo com os dados da pesquisa demonstrados na Tabela 1, em relação ao perfil sociodemográfico da amostra do estudo, $82 \%$ das puérperas tinham entre 18 e 20 anos. A maternidade é algo complexo e exige extrema dedicação da mulher, principalmente, quando vivenciada na adolescência e/ou quando essa responsabilidade é de uma única pessoa. Um grande desafio de ser mãe solteira é a pressão que a sociedade impõe sobre a ausência paterna, ou até mesmo, comentários sobre a falta de pulso masculino na educação de uma criança, estes que são considerados "normais" nessa sociedade. Segundo a OMS, a taxa de gestação na adolescência no Brasil é alta, com 400 mil casos/ano. A gestação, nesta fase, eleva a prevalência de complicações para a mãe e para o feto/recém-nascido.

Com base nos dados apresentados pelo IBGE (2020), o Brasil tem, aproximadamente, 11,4 milhões de mães solteiras, sendo mais da metade negras (7,5 milhões). Dentre todas as dificuldades, como a desigualdade social, a desigualdade salarial e a pressão que a sociedade impõe sobre a ausência paterna, como já foi abordado, vale ressaltar que as mães negras têm menos acesso aos serviços básicos do que as mães brancas. Esses dados confirmam a quão acentuada é essa questão de dupla jornada das mães solteiras e negras no Brasil. Essas mulheres, que chefiam famílias sozinhas, vão morar em lugares com falta de 
saneamento básico, devido a sua respectiva condição socioeconômica, e isso faz com que o processo da gestação/puerpério seja ainda mais delicado.

A presente pesquisa demonstra que $35 \%$ das entrevistadas concluíram o Ensino Médio, dentre as $65 \%$ que não concluíram, aproximadamente 50\% relataram que a gravidez foi um dos principais motivos pela desistência dos estudos. Moura et al. (2020), relatam, em sua pesquisa, que estudos analisaram que os fatores preditores da evasão escolar, com a experiência de gravidez, demonstram que 94,4\% das jovens afirmaram ter interrompido os estudos em algum momento da vida, durante ou após uma gravidez e 54,4\% abandonaram os estudos definitivamente. Com isso, podemos afirmar que a escolaridade é um dos determinantes sociais de saúde relacionado à gravidez na adolescência.

Com relação ao puerpério em mulheres negras, 70,5\% das entrevistadas se enquadram dentro desse parâmetro étnico. Em pesquisa realizada por Oliveira et al. (2020), o autor demonstra que 28,8\% da amostra do estudo é composta de puérperas que se autodeclaram negras. Segundo dados do Instituto Brasileiro de Geografia e Estatística - IBGE (2017), as mulheres brancas e com o Ensino Médio completo possuem menos filhos que mulheres autodeclaradas pretas e pardas, que portam um grau de escolaridade inferior. Como já foi citado, a escolaridade é um dos determinantes relacionados à gravidez na adolescência, por isso, as taxas de analfabetismo de pretos e pardos são maiores que o dobro da de brancos.

Tabela 2 - Porcentagem das puérperas entrevistadas segundo relatos sobre a amamentação. Tracunhaém- PE, USF Liberdade, 2021.

\begin{tabular}{|l|l|}
\hline Benefícios do leite materno nos primeiros 6 meses de vida & $\%$ \\
\hline Sim & $58 \%$ \\
\hline Não & $42 \%$ \\
\hline Total & $100 \%$ \\
\hline Importância da amamentação & \\
\hline Sim & $94 \%$ \\
\hline Não & $6 \%$ \\
\hline Total & $100 \%$ \\
\hline & \\
\hline Dificuldade para amamentar durante a pandemia de COVID-19 & $100 \%$ \\
\hline Não & $0 \%$ \\
\hline Sim & $100 \%$ \\
\hline Total & \\
\hline & \\
\hline Recebeu alguma orientação sobre o aleitamento em tempos de pandemia de \\
COVID-19
\end{tabular}

Fonte: Entrevista (2021)

De acordo com os dados expostos na Tabela 2, sobre a amamentação, 58\% das mães informaram saber quais os benefícios da amamentação exclusiva nos primeiros 6 meses de vida da criança e 94\% das entrevistadas informaram achar 
importante a amamentação. Sobre o ALM, durante a pandemia de COVID-19, 100\% das entrevistadas afirmaram não possuir dificuldades com a amamentação e $76 \%$ afirmaram que não receberam orientação dos profissionais da saúde sobre o aleitamento em tempos de pandemia de COVID-19.

O LM é o alimento adequado para as crianças nos primeiros meses de vida, tanto do ponto de vista nutritivo e imunológico, quanto no plano psicológico, além de favorecer o vínculo mãe-filho quando o ato de amamentar é bem vivenciado pelas mães. Segundo Eduardo (2015), recapitulando o que diz o Ministério da Saúde, a prática da amamentação salva a vida de 6 milhões de crianças por ano, prevenindo diarreia e infecções respiratórias agudas, além de ser responsável por cerca de um terço da diminuição da fertilidade observada nas últimas décadas.

De acordo com Brasil (2009), os benefícios do aleitamento materno são inúmeros, como diminuir a probabilidade do desenvolvimento dos processos alérgicos pelo retardo da introdução de proteínas heterólogas (leite de vaca) e pela ação provável de uma célula chamada macrófago, existente em grande quantidade no colostro; favorecer o desenvolvimento neuropsicomotor, melhorando, profundamente, a relação mãe-filho, com todos os benefícios consequentes; proteger a criança contra infecções, principalmente as relacionadas ao aparelho respiratório, que transcende o aspecto nutricional e ultrapassa a díade mãe-filho. Na realidade brasileira, devido aos altos índices de gravidez na adolescência associados à carência socioeconômico-cultural, é mais que necessário o incentivo ao ALM, como também a capacitação para as mães em como amamentar seu bebê exclusivamente, além de estimular a interação com seu filho na hora da amamentação.

Através desse estudo, podemos constatar que, apesar das puérperas saberem da importância do AME para o adequado crescimento e desenvolvimento da criança, observa-se que $76 \%$ das mães entrevistadas ainda desconheciam questões simples sobre a prática da amamentação em tempos de COVID-19, bem como as propriedades e funções do leite materno para a mãe e bebê. O baixo nível de conhecimento verificado e a escassez de informações, recebidas sobre amamentação, sinalizam para a urgência de um programa de orientação e promoção do ALM e orientação da gestante durante o pré-natal, em tempos de pandemia de COVID-19.

Ainda tendo como base Brasil (2009), o profissional de saúde tem como objetivo direcionar mães às práticas educativas em saúde para que elas sejam estimuladas a iniciar, ou continuar, amamentando as crianças, pois os benefícios do aleitamento materno superam, consideravelmente, os riscos potenciais de transmissão do novo coronavírus, priorizando, não só o aspecto fisiológico da lactação, como também o sociocultural, garantindo, assim, o sucesso do aleitamento materno.

\section{Considerações Finais}

Conforme os dados apresentados, a amamentação tem um papel fundamental para a saúde da criança, além de favorecer o vínculo entre mãe e filho. Observou-se também que a maior parte das participantes da pesquisa desconhecia os benefícios relacionados ao aleitamento materno. Segundo a pesquisa, a maioria das puérperas não recebeu orientação quanto aos cuidados durante a amamentação no período da pandemia, podendo, assim, aumentar o risco de contaminação da criança pelo vírus SARS-CoV-2.

Portanto, sugerimos que em trabalhos futuros, a temática possa ser abordada de forma ainda mais profunda, destacando pontos que são necessários e que, até então, não têm tanta visibilidade. Apesar de ser um assunto de grande relevância, é algo que precisa ter mais estudos relacionados, apresentando perspectivas diferentes sobre o tema proposto.

\section{Referências}

Brasil. Ministério da Saúde: Biblioteca Virtual em Saúde. (2009). 01 a 08/02 - Semana Nacional de Prevenção da Gravidez na Adolescência. Editora do Ministério da Saúde. https://bvsms.saude.gov.br/01-a-08-02-semana-nacional-de-prevencao-da-gravidez-na-adolescencia/.

Brasil. Ministério da Saúde. (2009). Secretaria de Atenção à Saúde. Departamento de Atenção Básica. Saúde da criança: nutrição infantil: aleitamento

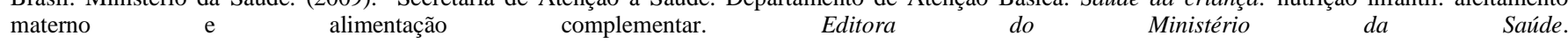


https://bvsms.saude.gov.br/bvs/publicacoes/saude_crianca_nutricao_aleitamento_alimentacao.pdf .

Brasil. Universidade Aberta do Sistema Único de Saúde (UNA-SUS). (2020). OMS - perguntas e respostas sobre COVID-19, gravidez, parto e amamentação. Editora do Ministério da Saúde. https://www.unasus.gov.br/noticia/oms-perguntas-e-respostas-sobre-covid-19-gravidez-parto-e-amamentacao.

Eduardo, E. F. A. F. (2015). Um olhar sobre a arte de amamentar. 30 f. Trabalho de Conclusão de Curso (Especialização em Saúde da Família) -

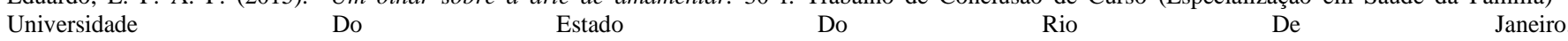
https://ares.unasus.gov.br/acervo/html/ARES/8061/1/Eveline\%20de\%20F\%C3\%A1tima\%20Almeida\%20Fonseca\%20Eduardo.pdf.

Fiocruz - Fundação Oswaldo Cruz. (2020). OMS - Benefícios da amamentação superam riscos de infecção por COVID-19. Editora Fiocruz. https://www.bio.fiocruz.br/index.php/br/noticias/1963-oms-beneficios-da-amamentacao-superam-riscos-de-infeccao-por-covid-19.

Fonseca, R.M. S. et al. (2021). O papel do banco de leite humano na promoção da saúde materno infantil: uma revisão sistemática. Revista Ciência \& Saúde Coletiva, Viçosa, v. 26, n.1, p. 309-318. https://www.scielo.br/j/csc/a/JVy96MGzR7gwDn57kTP46js/?lang=pt.

Ibge - Instituto Brasileiro de Geografia e Estatística - Censo Agro (2017). Mulheres mais escolarizadas são mães mais tarde e têm menos filhos. Rio de Janeiro. https://censos.ibge.gov.br/2013-agencia-de-noticias/releases/13899-asi-sis-2010-mulheres-mais-escolarizadas-sao-maes-mais-tarde-e-tem-menosfilhos.html.

Ibge. Desigualdades Sociais por Cor ou Raça no Brasil. (2020). https://www.ibge.gov.br/estatisticas/sociais/populacao/25844-desigualdades-sociais-por-corou-raca.html?=\&t=sobre.

IFF-FIOCRUZ. (2020). Instituto Nacional de Saúde da Mulher, da Criança e do Adolescente Fernandes Figueira. (COVID-19 e Aleitamento Materno: orientações do MS, SBP e rBLH. Rio de Janeiro. https://portaldeboaspraticas.iff.fiocruz.br/atencao-crianca/covid-19-e-aleitamento-materno-orientacoes-dasbp-e-rblh/.

Lüdke, M; André, M. (2013). Pesquisa em Educação: abordagens qualitativas. São Paulo: EPU, 1986.

Martins, M. M. et al. (2021). Características clínicas e laboratoriais da infecção por sars-cov-2 em crianças e adolescentes. Revista Paulista de Pediatria, v. 39. https://www.scielo.br/j/rpp/a/tPC9RLntm6sSjVFBLWFdKnf/?lang=pt.

Melo, L. P.C. et al. (2020). Aleitamento materno em tempos de covid-19: uma revisão integrativa. Research, Society and Development, v. 9, n. 9. https://www.rsdjournal.org/index.php/rsd/article/view/7074.

Moura, F. S. et al. (2021). Determinantes sociais da saúde relacionados à gravidez na adolescência. Revista de Saúde Pública do Paraná, Paraná, v. 4, n. 1, p. 133-150. http://revista.escoladesaude.pr.gov.br/index.php/rspp/article/view/452/201.

Oliveira, J. V. L. et al. (2021). Estratégia de saúde da família:Problemas com a saúde da mãe e do bebê causados pelo não cumprimento do esquema vacinal. Research, Society and Development, [S. l.], v. 10, n. 3. https://rsdjournal.org/index.php/rsd/article/view/13772/12336.

OPAS - Organização Pan-Americana da Saúde; OMS - Organização Mundial da Saúde. Folha informativa sobre COVID-19. Brasília, [20-?]. https://www.paho.org/pt/covid19.

Pereira, A. S. et al. (2018).Metodologia da pesquisa científica. UFSM. $1 . \quad$ ed. E-book. Santa Maria. https://repositorio.ufsm.br/bitstream/handle/1/15824/Lic_Computacao_MetodologiaPesquisa-Cientifica.pdf?sequence=1.

Portugal, Sociedade Portuguesa Neonatologia. (2020). Recomendações para a abordagem do recém-nascido em contacto com a infeção por SARS-CoV-2 (COVID19). Lisboa. http://www.spneonatologia.pt/wpcontent/uploads/2020/03/COVID-19_170320.pdf.

Silva, F. C. et al. (2021). Isolamento social e a velocidade de casos de covid-19: medida de prevenção da transmissão. Revista Gaúcha de Enfermagem, v. 42, n. 1. https://preprints.scielo.org/index.php/scielo/preprint/view/1509/2392.

Tacla, M. T. G. M. et al. (2020). Reflexões sobre o aleitamento materno em tempos de pandemia por COVID-19. Revista da Sociedade Brasileira de Enfermeiros Pediatras, v. 20, n. 1, p. 60-76. https://journal.sobep.org.br/wp-content/uploads/articles_xm1/2238-202X-sobep-20-spe-0060/2238-202X-sobep20-spe-0060.x19092.pdf. 